\title{
ILCEA
}

Revue de l'Institut des langues et cultures

d'Europe, Amérique, Afrique, Asie et Australie

27 | 2016

Approches ergonomiques des pratiques

professionnelles et des formations des traducteurs

\section{La traduction comme travail : perspectives croisées en ergonomie, sociologie et traductologie}

Translation as Work: Cross Perspectives in Ergonomics, Sociology and

Translation Studies

\section{Anna Kuznik}

\section{(2) OpenEdition}

Journals

Édition électronique

URL : http://journals.openedition.org/ilcea/4036

DOI : 10.4000/ilcea.4036

ISSN : 2101-0609

Éditeur

UGA Éditions/Université Grenoble Alpes

Édition imprimée

ISBN : 978-2-84310-336-0

ISSN : $1639-6073$

\section{Référence électronique}

Anna Kuznik, «La traduction comme travail : perspectives croisées en ergonomie, sociologie et

traductologie », ILCEA [En ligne], 27 | 2016, mis en ligne le 08 novembre 2016, consulté le 19 avril 2019. URL : http://journals.openedition.org/ilcea/4036 ; DOI : 10.4000/ilcea.4036

Ce document a été généré automatiquement le 19 avril 2019

(C) ILCEA 


\section{La traduction comme travail : perspectives croisées en ergonomie, sociologie et traductologie}

Translation as Work: Cross Perspectives in Ergonomics, Sociology and

Translation Studies

Anna Kuznik

\section{Introduction}

1 Le titre du présent article s'inspire de deux ouvrages de Daniel Gouadec: le premier publié en français en 2002 sous le titre Profession: traducteur, et le deuxième publié en 2007 en anglais sous le titre Translation as a Profession. Nous étudierons la profession de traducteur sous l'angle du travail, ce dernier constituant une perspective relativement novatrice en traductologie et étant par ailleurs devenu l'objet d'études de plusieurs disciplines scientifiques, appelées collectivement sciences du travail.

2 L'objectif de cet article est de montrer comment trois disciplines connexes, à savoir l'ergonomie organisationnelle, la sociologie du travail et la traductologie, ont contribué à la création de notre nouveau projet de recherche, au niveau de la conception de l'objet d'étude, de la méthodologie de recherche et des conditions de travail sur le terrain.

3 L'ergonomie organisationnelle, présentée dans la section 2 du présent article, vise à décrire les aspects organisationnels des pratiques de travail afin d'optimiser le bien-être des personnes et la performance des systèmes.

4 La sociologie du travail s'intéresse au caractère social de la production et à la collaboration des collectifs dans les milieux de travail. Les études effectuées dans le cadre de la sociologie du travail s'apparentent aussi aux études organisationnelles qui apportent un cadre méthodologique aux procédures de collecte de données au sein des entreprises. Ces considérations méthodologiques sont typiques des méthodes observationnelles, non expérimentales, dans lesquelles le chercheur ne manipule pas de 
variables indépendantes mais se concentre sur les variables dépendantes, représentées par des indicateurs dont la collecte s'effectue moyennant différentes techniques d'observation. Des restrictions spéciales propres aux études effectuées au sein des organisations (et plus précisément, au sein des entreprises) viennent s'y ajouter. Ces deux disciplines (la sociologie du travail et les études organisationnelles) sont présentées dans la section 3 du présent article.

5 La traductologie étudie, entre autres, les métiers et les conditions de production des activités et des services de traduction. Dans la section 4, nous présentons notre propre trajet de recherche afin de cerner le contenu du travail des traducteurs internes dans des schémas organisationnels différenciés (petite agence de traduction, entreprise de traduction, PME industrielle), ce contenu du travail étant conçu comme ensemble d'activités organisées autour d'un axe temporel, enchaînées dans un processus productif et propres à un poste de travail.

6 L'ensemble de ces réflexions préliminaires nous aidera à élaborer un nouveau projet de recherche qui porte sur la configuration des services de traduction et services annexes dans des entreprises de traduction à Grenoble (France, phase pilote) et à Wrocław (Pologne, étude principale).

\section{L'ergonomie appliquée à l'étude des pratiques de traduction : état de l'art}

7 C'est au cours du colloque "Traduction et Ergonomie", organisé en octobre 2010 à l'université Stendhal de Grenoble ${ }^{1}$, que l'ergonomie a été proclamée comme "un autre cadre théorique [...], un nouveau paradigme qui permettrait d'interpréter la réalité des métiers de la traduction aujourd'hui et dans les vingt ans à venir " (Lavault-olléon, 2011b: 5 ; voir aussi Lavault-Olléon, 2011a). Ce nouveau paradigme a permis de se pencher, avec une vision renouvelée, sur la relation entre le traducteur et ses moyens, méthodes et milieux de travail (Lavault-Olléon, 2011b : 5), le traducteur étant redéfini en termes « d'ingénieur en communication multilingue et multimédia » (Gouadec, 2002) ou «de personnel assistant multilingue " et "d'expert en communication multilingue » (Kuznik, 2016 : 227).

8 La définition de l'ergonomie formulée en 2000 par l'IEA (International Ergonomics Association) présente l'ergonomie comme une discipline scientifique qui vise à comprendre les " interactions entre les humains et les autres composantes d'un système " mais aussi comme « la profession qui applique principes théoriques, données et méthodes en vue d'optimiser le bien-être des personnes et la performance globale des systèmes " (Lancry, 2009: 20, cité dans Lavault-Olléon, 2011b: 5). L'objectif de l'ergonomie est double : (1) identifier les aspects problématiques, conflictuels, dangereux des systèmes de travail, dont le travailleur constitue le point central, et (2) proposer, élaborer et appliquer des solutions, des remèdes, des améliorations efficaces, en vue d'optimiser le bien-être des individus (sécurité, santé, confort, satisfaction, facilité d'usage des objets) ainsi que la performance des systèmes de production (efficacité, productivité, fiabilité, durabilité). L'approche holistique de l'homme, "où celui-ci est simultanément pensé dans ses dimensions physiologiques, cognitives et sociales » (Falzon, $2004: 20)$ a donné naissance à trois branches connues de cette discipline: l'ergonomie physique, cognitive et organisationnelle. Les méthodes de l'ergonomie comprennent une double dimension: (1) 
l'observation et l'analyse des situations réelles du travail pour pouvoir les décrire et ainsi identifier des conflits et points de friction; et (2) l'action d'optimisation, c'est-à-dire la mise en place des améliorations pour pouvoir remédier à ces conflits émergents (LavaultOlléon, 2011b: 5 et 7).

De nombreuses recherches effectuées dans le cadre des recherches traductologiques se sont déjà rapprochées des intérêts et des méthodologies de travail propres à l'ergonomie. Le numéro 14 de la revue ILCEA (Lavault-Olléon, 2011c) issu du colloque «Traduction et Ergonomie ", en donne de bons exemples.

Les études traductologiques qui s'inscrivent dans l'approche ergonomique organisationnelle dont l'objectif est «l'optimisation des systèmes sociotechniques, incluant leur structure organisationnelle, règles et processus " (Falzon, 2004: 19), sont déjà nombreuses et abordent une problématique vaste et complexe qui englobe le travail de traduction «multiforme, multilingue et multimédia, mais également collaboratif et évolutif » (Lavault-Olléon, 2011b : 5), ses différentes formes et processus, ses composantes (tâches variées et multiples), les outils de travail mis en jeu (outils informatiques, principalement), les acteurs des processus de production (collègues et supérieurs) ainsi que les schémas organisationnels, par exemple le schéma circulaire centré sur le traducteur, le schéma linéaire dans lequel le traducteur est un maillon d'une chaîne, ou le schéma de type réticulaire (Lavault-Olléon, 2011b: 10). À cela s'ajoutent les temps, rythmes et cycles de travail, les normes (entre autres, normes ISO et CEN 15038), la qualité du service de traduction livré et la relation au travail dans son ensemble, dans un sens très large (les missions et l'identité professionnelles, l'identification du groupe professionnel aux contenus du travail effectué, notamment).

11 Les communications présentées au colloque «Traduction et Ergonomie » de 2010 qui étaient proches de l'ergonomie organisationnelle se sont centrées sur l'ergonomie du poste de travail idéal dans la perspective de chacun des acteurs de la chaîne de traduction: traducteur, entreprise de traduction et donneur d'ouvrage (Toudic \& Brébisson, 2011), sur les fonctions qui doivent impérativement être présentes dans un logiciel de mémoire de traduction (Grass, 2011), sur les critères qui fondent la qualité ergonomique d'un document technique relevant de l'aéronautique (Ryan, 2011), sur le rôle clé que peut jouer un traducteur dans une équipe pluridisciplinaire en faisant valoir son expertise linguistique et interculturelle et en assumant son rôle créatif dans le cas de l'adaptation publicitaire (Vandal-Sirois, 2011), ou encore sur l'identité professionnelle des traducteurs, majoritairement des prestataires de services indépendants, en tant que groupe sociologique au nord du Portugal (Ferreira-Alves, 2011).

Le colloque suivant, intitulé "Traducteurs à l'œuvre: approches ergonomiques des pratiques professionnelles et des formations de traducteurs", qui s'est tenu les 5 et 6 mars 2015 toujours à Grenoble, a comporté deux interventions s'inscrivant directement dans l'ergonomie organisationnelle (Foedisch, 2015; Kuznik, 2015) ainsi que des interventions abordant la très vaste problématique des aides, des outils informatiques et des ressources accessibles en ligne.

13 Le texte même de l'appel à contributions du colloque de 2015, en évoquant des notions telles que l'activité du traducteur au travail, l'organisation des tâches, les processus de travail, les contraintes organisationnelles, la productivité et les coûts, les conditions de travail, le bien-être, l'identité, l'autonomie et la responsabilité des traducteurs, l'impact des technologies, les nouveaux modes de collaboration, a suscité toutes sortes de réflexions organisationnelles de type ergonomique autour de la "chaîne documentaire ", 
$\mathrm{du}$ « processus d'émission de documents multilingues, qui s'intègre lui-même au vaste système de production et de gestion électronique des documents" (Lavault-olléon, $2011 \mathrm{~b}$ : 9), dans lequel la « place de la traduction [...] devient moins autonome car intégrée au processus global de production de contenu » (Lavault-Olléon, 2011b: 9; voir aussi Kuznik, 2016).

Ces développements théoriques et ces propositions de mesures d'amélioration, dans le cadre de l'ergonomie organisationnelle et de la traductologie, n'auraient pas été possibles sans l'application d'une méthodologie aussi rigoureuse que créative, s'appuyant principalement sur «l'observation des comportements au travail, les enquêtes sur le terrain et les entretiens avec les intéressés » (Lavault-Olléon, 2011b: 7), méthodologie commune pour ces deux disciplines mais aussi pour une troisième, ciblée sur l'observation des situations réelles du travail, à savoir la sociologie du travail.

\section{La sociologie du travail : observation des situations de travail réelles}

«I like work: it fascinates me. I can sit and look at it for hours. » Cette phrase humoristique, formulée par Jerome K. Jerome dans le chapitre 15 de son livre de 1889, Three Men in a Boat (Jerome, 2006: 49) reflète l'importance du travail comme objet d'étude et d'observation des sociologues dès les débuts de la sociologie du travail. Comme chaque sous-discipline sociologique, cette science s'intéresse à l'articulation et à la distribution du travail dans les différents groupes socio-professionnels. Bien qu'elle soit dépourvue de l'intérêt immédiat d'optimisation du travail, propre à l'ergonomie, elle est une sous-discipline nettement critique, comme l'est d'ailleurs toute «bonne » recherche sociologique (Mills, 1987). Cette vision critique de la sociologie du travail permet d'identifier les conflits sousjacents et les points faibles des systèmes et des groupes socio-professionnels, qui peuvent être, par la suite, améliorés par des disciplines à vocation plus technique.

\section{Fondement de la discipline : les premières études sociologiques dans le secteur industriel}

L'émergence des études qui utilisaient la technique d'observation directe du travail dans l'industrie juste après la seconde guerre mondiale a probablement été une conséquence du mélange des classes sociales (intellectuelles et ouvrières) dans les usines et du plein emploi dans une période très dynamique de reconstruction de pays entiers et de leurs ressources industrielles (Peneff, 1998: 5). C'est à cette époque-là qu'il faut situer la naissance en France de la sociologie du travail. Cette discipline étudiait les conditions du travail directement à travers la participation aux processus de production. C'est alors, dans les années 1950, que sont apparues les recherches variées des jeunes sociologues réunis autour de Friedmann: Frisch-Gautier, Peyre, Dofny et Aumont. Dans la décennie 1960-1970, de manière isolée et en s'appuyant sur des cadres théoriques assez diversifiés, d'autres chercheurs (Sainsauliau, Linhart, Bernoux, Motte \& Saglio) ont mené des études sur le travail ouvrier. Ensuite Durand, Prestat et Willemer, au début des années 1970 ont analysé les postes de travail dans le secteur sidérurgique.

Parallèlement, autour de 1950, un groupe de chercheurs nommé "groupe de Manchester » s'est constitué aux États-Unis autour de l'analyse du secteur industriel. L'un 
de ses représentants, Gluckman, un anthropologue africaniste, a orienté la recherche de ses étudiants vers l'ethnographie du travail. Les études de Lupton, qui datent du début des années 1960 sur le travail dans les usines d'équipement électrique et les usines de plastique se situent dans le même courant.

Il faut mentionner aussi la contribution de Roy (1952), un ouvrier à la chaîne qui a calculé sa propre productivité à l'insu de ses supérieurs afin de découvrir à quel rythme journalier lui et ses collègues de travail surpassaient ou limitaient les normes et les cadences attendues, dans le but d'assurer un profit maximum en fonction des dépenses physiques et nerveuses qu'ils seraient capables de supporter. Cette étude a probablement été le premier cas d'auto-observation dans un contexte de production tayloriste.

Sur le terrain européen, un sociologue français, Terssac (1992; voir aussi Kuznik, 2010 : 105-108), a concentré ses recherches sur les processus continus automatisés de l'industrie (secteurs chimie, cimenterie, électricité et imprimerie). En observant des situations de travail réelles, ce chercheur a essayé de modéliser le système social existant à l'intérieur de ces usines : modes d'organisation réellement mis en œuvre par les collectifs humains employés à la production, et modes de coopération afin de créer des règles de production plus efficaces.

\section{Secteur tertiaire. Principales études et métiers observés}

20 Étant donné le développement extrêmement accéléré du secteur tertiaire, les métiers liés aux activités de services demandent une attention spéciale dans la recherche sociologique, d'autant plus que ce secteur reste très diversifié, avec des limites floues (Durand, 2004 : 207-251; Kuznik, 2014 ; Peneff, 1998 : 16).

21 L'analyse des situations de travail réelles effectuée par Durand $(2000,2004)$ concerne les notions de flux tendu et d'implication contrainte dans le secteur automobile et dans les services. Ce chercheur étudie en premier lieu les aspects organisationnels de la production maigre, allégée, sans gaspillage («lean production»), en flux tendu («just on time ») et ensuite son impact psychologique sur les individus. D'après Durand, ce système de fabrication élimine le besoin d'accumuler des stocks, et de cette manière, étire le flux de production. Le travailleur impliqué dans cette chaîne de fabrication s'autodiscipline pour assurer le fonctionnement d'une production toujours tendue à l'extrême. Ces circonstances de travail créent bien évidemment chez l'individu une forte charge mentale, émotionnelle et intellectuelle, mais il l'accepte de lui-même.

Les hôpitaux, organisés en bureaucraties professionnelles (Kuznik, 2011: 288), ont eux aussi fourni aux sociologues bien des sujets de recherches. Peneff (1992) mentionne une étude motivée par une fréquente critique formulée par les supérieurs hiérarchiques envers les infirmières : apparemment celles-ci n'étaient pas assez attentives, réceptives et ouvertes vis-à-vis des patients. Afin de saisir le conflit voilé entre les médecins et les infirmières, le chercheur a démontré le poids réel, la fonction et l'importance des rapports écrits sur les patients (fonction administrative remplie par les infirmières), et l'invasion de cette fonction administrative au détriment de la fonction sanitaire du personnel hospitalier.

Cicourel (2002) situe ses études sociologiques dans un service hospitalier de consultations externes spécialisées. Il analyse les échanges verbaux entre Sari, l'infirmière, et tout le reste du personnel du service. Cette étude met en relief les conditions de travail en 
situation de surcharge cognitive, conséquence d'interruptions constantes et de l'irruption de tâches réalisées en parallèle. Sari s'adapte à ces conditions en ayant recours à des supports externes pour sa mémoire à court terme (les «post-it » et l'ordinateur). Cicourel révèle en outre le manque de symétrie dans les relations entre l'infirmière et les médecins.

Verd, Barranco et Moreno (2007) ont également mené une recherche sur le contenu du travail $\mathrm{du}$ personnel administratif dans un service de consultations externes (traumatologie et dermatologie) et interne (stérilisation) d'un hôpital catalan. Leur étude s'inscrit dans le cadre théorique d'une activité située et de la cognition socialement distribuée (Lozares, Verd \& Massó, 2004). Les chercheurs ont pu identifier plusieurs processus de travail, centraux et secondaires, et les exigences très sévères imposées par ces postes de travail (travail continu sans pauses, superposition de tâches, complexité mentale et émotionnelle des activités, surcharge mentale, flux tendu et implication contrainte du personnel).

Cicourel (2002: 4) souligne que très peu d'études sociologiques s'effectuent au sein des bureaucraties professionnelles, même si certains chercheurs ont pu accéder à des organisations particulièrement inaccessibles (Peneff, 1998), comme par exemple les prisons, pour y étudier les missions des fonctionnaires, et les écoles publiques pour analyser la mesure du temps réel dédié à l'enseignement des langues et des mathématiques.

Une série d'autres métiers du secteur tertiaire a également intéressé les sociologues du travail : les secrétaires de bureaux, les caissières de supermarchés et les serveuses de restaurants (Peneff, 1998).

\section{Caractéristiques méthodologiques de la recherche menée au sein des organisations}

27 Les études effectuées dans le cadre de la sociologie du travail s'apparentent aussi aux études organisationnelles (Coller \& Garvía, 2004; Rosenstiel, 2004) par les difficultés particulières inhérentes à la recherche menée sur le terrain au sein d'organisations : entreprises et institutions. L'ensemble des études basées sur l'observation dans les organisations présentent, de façon plus ou moins prononcée, les caractéristiques méthodologiques qui suivent.

En premier lieu, ce sont des études interdisciplinaires par définition parce que les organisations ont elles-mêmes une nature très complexe et multidimensionnelle (Rosenstiel, 2004 : 130).

29 En deuxième lieu, il est fortement recommandé d'utiliser pour ces études une logique méthodologique abductive, c'est-à-dire une alternance d'induction et de déduction. Généralement, les étapes inductive et déductive correspondent à deux niveaux de mesure différents. Peneff (1998: 16) mentionne un premier niveau, plus fondamental, qui répond aux questions : qui ?, quand?, combien ?, où ? Ce niveau envisage les caractéristiques des acteurs (sexe, âge, profession, statut) et des actions (lieu, durée, nombre d'interventions). Les indicateurs de mesure du premier niveau seraient liés au nombre de décisions prises, aux nouvelles et anciennes tâches, aux perturbations et pauses. Ils seraient également liés au nombre d'accidents et d'urgences, aux objectifs atteints par rapport aux objectifs prévus, au temps passé en réunions, à la fréquence des conflits et aux sujets de 
discussions internes. Le second niveau de mesure est beaucoup plus complexe car il aborde des interprétations des acteurs concernant ces situations de travail mais également des caractéristiques intrinsèques des actions ainsi que leur valeur symbolique (Peneff, 1998 : 11).

En troisième lieu, les études observationnelles effectuées au sein des entreprises s'inscrivent souvent dans le paradigme méthodologique des méthodes mixtes. Verd et López (2008: 13) définissent ce paradigme d'une manière très ample comme la combinaison des techniques quantitatives et qualitatives dans une même étude.

31 En quatrième lieu, comme conséquence directe de la grande difficulté d'accès aux entreprises, principalement à cause du caractère confidentiel de l'information, les études observationnelles effectuées en interne sont des études de cas. Cette difficulté d'accès a un impact sur la procédure d'échantillonnage et sur le degré de représentativité de l'échantillon par rapport à la population. Plus l'étude est superficielle, plus simple est l'accès à une entreprise, et l'étude peut alors porter sur davantage d'unités (entreprises) qu'une étude plus poussée. Coller et Garvía (2004: 101) signalent que généralement, les études portent sur une seule ou un très petit nombre d'organisations à la fois, car les coûts de la recherche sont ainsi réduits et les ressources plus restreintes (outils, personnel, infrastructure). La richesse d'une étude de cas ne découle pas du nombre d'entreprises visitées, mais de la variété et de la combinaison des hypothèses de recherche (Coller \& Garvía, 2004 ; voir aussi Flyvbjerg, 2004).

32 Finalement, en cinquième lieu, les études observationnelles effectuées au sein des entreprises partagent la même difficulté d'accès aux unités d'analyse. Dans l'approche de la sociologie du travail, Durand (2004 : 254-259) distingue trois niveaux d'entreprises : (1) l'entreprise normative qui dit ce que doit être l'entreprise; (2) l'entreprise normée, visible, celle que l'on visite et étudie ; (3) l'entreprise simulée, invisible, qui se cache ou qui ne veut pas être vue. Les études de cas, grâce à leur vision étendue de l'objet d'étude, permettent aux chercheurs d'accéder à ce troisième niveau, le plus authentique et le plus intéressant. Dans la recherche traductologique, de nombreux auteurs ont mentionné la difficulté d'accès et la difficulté de collaboration avec les entreprises, parmi lesquelles les sociétés de traduction (Ehrensberger-Dow, 2014 ; Kuznik, 2010 : 108-109 ; Risku, 2014).

Cette difficulté est accrue surtout dans le cas du recours à l'observation directe des lieux de travail. Peneff (1998: 15) souligne l'existence de cette difficulté spécialement dans les études observationnelles menées dans le secteur tertiaire, à cause de la proximité physique et symbolique des acteurs soumis à l'observation: il n'est pas possible d'effectuer une observation directe d'une secrétaire ou d'une infirmière sans observer, même indirectement, le directeur ou le médecin au travail. C'est de là que vient la réticence des entreprises à laisser entrer les chercheurs. L'observation effectuée, les résultats obtenus et les conclusions formulées touchent indirectement la hiérarchie du pouvoir des organisations à but lucratif. Ce phénomène se présentait beaucoup moins dans les études observationnelles menées dans l'industrie, où les ouvriers et les cadres étaient physiquement plus séparés.

L'ensemble de ces caractéristiques doit être pris en compte lors de la conception et pendant la réalisation des études observationnelles empiriques qui portent sur les modalités sociales du travail dans les entreprises et chez les fournisseurs de services, qui incluent les services langagiers. 


\section{La sociologie du travail appliquée à l'étude de la traduction. Étude du contenu du travail des traducteurs internes}

la production des services de traduction pour et en collaboration avec différents acteurs de l'entreprise (Gouadec 2002, 20015a, 2005b, 2007 ; Kuznik, 2014), cette activité apparaît entourée, dans le processus de production, d'autres activités annexes mais indispensables. D'après le modèle d'exécution de la prestation de traduction de Gouadec (2002: 19, 2005a, 2005b, $2007: 15)$, plusieurs activités précèdent la traduction proprement dite (activités en amont du processus), et une chaine d'activités annexes à la traduction se situe en aval. Dans cette vision productive, l'activité de traduction reste au centre du processus de production, donnant sens à la mission globale de la prestation fournie (image de marque, ancrage des services de consultation linguistique dans la compétence de traduction, notamment) et à l'entité qui s'en charge (traducteur indépendant, département interne de traduction, société de traduction, entre autres). Cependant, dans la plupart des cas, la traduction constitue une activité externalisée et dès lors physiquement absente de cette entité (Kuznik, 2010: 261, 340-347, 426-429), d'où sa position résolument paradoxale (Gouadec, 2007 ; Kuznik, $2010: 440$ ). La sous-traitance en de longues chaînes de sous-traitants consécutifs ajoute à son tour une tâche très importante aux processus de production du service, à savoir la gestion de la soustraitance (Kuznik \& Verd, 2010).

ILCEA, 27| 2016 
38 Il existe par ailleurs de nombreuses réflexions et de nombreux témoignages qui rendent compte de différentes dimensions et de différents rôles des traducteurs, y compris des traducteurs indépendants, dans les entreprises, aussi bien dans une approche diachronique (Skibińska \& Blumczyński, 2009) que synchronique (Lebtahi \& Ibert, 2004 ; Vandal-Sirois, 2011) en montrant les métiers des traducteurs dans toute leur complexité et richesse.

39 Ces deux réalités (la sous-traitance de la traduction et la variété des profils professionnels des traducteurs) nous ont amenée à nous poser la question du contenu réel du travail des traducteurs internes. Concrètement, nous nous sommes demandé quelles activités sont réellement effectuées sur les postes de travail des traducteurs internes, et dans quelles proportions respectives.

\section{Cadre conceptuel et méthodologique}

40 Pour répondre à cette question, nous avons commencé par situer ce nouvel objet d'étude, à savoir le contenu du travail entendu comme l'ensemble des activités réellement effectuées à un poste de travail, dans la discipline traductologique, en le rattachant au processus organisationnel de prestation de la traduction, celui-ci étant la concrétisation du processus de communication (Mayoral, 2001 : 48-49; voir aussi Kuznik, 2008 : 369-371).

41 Nous avons ensuite défini du point de vue théorique la notion de tâche de traduction (et d'interprétation) en tant qu'unité minimale, de base et de référence, pour l'étude empirique de ce contenu (Kuznik, 2007a, 2008, 2010). Les tâches de traduction et d'interprétation seraient ainsi "des catégories observables directement, de type comportemental, propres à la performance de la personne qui réalise des traductions et des interprétations dans son milieu de travail (dans un cadre professionnel), qui tirent leur origine d'une compétence professionnelle non observable directement " (Kuznik, 2007a: 129), et dont les caractéristiques seraient les suivantes: (1) manifestations observables d'une compétence au niveau du comportement ; (2) noyaux organisateurs du travail ; (3) «graduables» de la simplicité à la complexité (difficulté crescendo); (4) orientées vers la production d'un service de traduction, de préférence, de qualité ; (5) se combinant avec « des tâches non propres à la traduction, mais similaires ou différentes " (Kuznik, 2007a : 129; voir aussi 2008: 374). Les tâches de traduction et d'interprétation pourraient être abordées suivant trois approches: (1) dans une perspective traductologique et psychologique, en tant que manifestations des compétences latentes; (2) dans une perspective productive, en tant qu'étapes d'un processus de travail et de production de service; et (3) dans une perspective socioprofessionnelle, en tant que composantes propres à des postes de travail concrets (Kuznik, 2008: 374-375). À cette étape de la recherche, qui a été menée principalement en langue espagnole, nous avons adopté le terme " tâche », traduction littérale du terme espagnol « tarea ».

Lavault-Olléon (2011b) apporte une précision concernant la distinction faite dans les approches ergonomiques entre " tâche » et " activité ». Suivant les ergonomes, une tâche est « pensée, prescrite et attendue par le commanditaire » et une activité « est la réponse que l'individu met en œuvre pour réaliser la tâche » et «se construit [...] sur la base d'interactions locales, dans un contexte et des circonstances matérielles et particulières " (Lavault-Olléon, 2011b: 6). Cette distinction entre «tâche » et «activité » fait écho aux trois niveaux d'entreprises mentionnés par Durand (2004: 254-259; entreprise normative, normée et simulée), présentés avant dans cet article. Pour nos bases 
théoriques d'étude du contenu du travail, une "tâche de traduction et d'interprétation ", vue dans sa réalisation, sa matérialisation et dans son contexte situationnel, est évidemment à comprendre comme une « activité » pour les ergonomes.

Par ailleurs, nous nous sommes penchés sur les possibilités méthodologiques permettant de mesurer cet objet d'étude, tout en étant consciente que les méthodes de mesure influencent forcément les formats de l'information empirique collectée, et vice versa. À cette étape, nous avons identifié dans les études traductologiques quatre indicateurs (formats de données empiriques) qui permettent de mesurer le contenu du travail des traducteurs internes, et qui, en outre, rendent compte du caractère très hétérogène de ce contenu, à savoir : (1) l'augmentation des postes de travail hybrides de traducteurs dans des offres d'emploi ; (2) la mesure qualitative et quantitative de l'impact réel de l'activité de traduction proprement dite dans la journée de travail et par poste de travail ; (3) la superposition ou le mélange de traduction et de gestion des processus de traduction; et (4) la nature hétérogène des stages de traduction (Kuznik, 2011).

Deux remarques importantes doivent tout de même être formulées quand on se propose d'étudier le contenu du travail entendu comme l'ensemble des activités réellement effectuées sur un poste de travail.

La première est une précision: le contenu réel d'un poste de travail dépend de la structure organisationnelle de l'entité qui l'englobe (Kuznik, 2011: 285-288), aussi appelée «schéma organisationnel» dans l'approche ergonomique organisationnelle (Lavault-Olléon, 2011b).

La deuxième apparait sous forme d'avertissement: la notion structurelle de poste de travail devient de plus en plus problématique avec le développement des économies mondiales, et notamment sous l'impact de la globalisation, de l'utilisation des technologies à grande échelle, et des changements entraînés par les formes flexibles d'organisation du travail (Kuznik, $2011: 288-290,2014: 9-10$ ).

\section{Paramètre « temps » et observation dans différents contextes organisationnels}

47 En nous appuyant sur des approches propres à la sociologie du travail (Verd, Barranco \& Moreno, 2007) pour développer nos intérêts traductologiques, nous avons choisi le paramètre structurant "temps", qui est l'un des paramètres structuraux les plus continus (au sens mathématique du terme) des études empiriques car il organise de manière optimale le contenu du travail des traducteurs internes, et par là même, il est susceptible de fournir des mesures fiables. Le choix d'un axe temporel reflète au fond notre conviction que nous sommes en réalité ce que nous faisons dans le temps de nos vies, dans le temps de nos journées de travail (et non pas ce que nous croyons faire, ou devrions faire, ou prétendons faire).

Ce premier choix a eu un impact direct sur celui des techniques de collecte des données, qui ont été principalement les techniques d'observation dans le temps. En utilisant ces techniques, nous avons étudié le contenu du travail des postes de traducteurs internes dans trois différents contextes organisationnels (quatre différents profils professionnels) : une petite agence de traduction polonaise (réinterprétation des données empiriques collectées par Stelmach au moyen d'une grille d'auto-observation; Kuznik \& Verd, 2010) ; dix entreprises de traduction de Barcelone (avec une grille d'observation 
directe ; Kuznik, 2010) ; une étude pilote menée à Rennes en 2007 avec quatre entreprises de traduction (Kuznik, 2007b) ; et une PME industrielle polonaise fabricant des machines pour le secteur alimentaire (analyse des prises de contact téléphoniques et par messagerie électronique; Kuznik, 2016). Les résultats de ces trois études ont été rassemblés sous forme de listes des activités effectuées par les traducteurs internes, avec leur distribution (en occurrences ou en pourcentages) dans une journée de travail type, afin d'en produire une vraie "image», une "photographie». Cette image ethnographique du contenu du travail des traducteurs internes apporte un argument empirique pour concevoir leur identité professionnelle d'une manière très vaste, complexe et hétérogène, et la rapproche d'autres métiers à forte composante intellectuelle dans le secteur des services au sein de schémas organisationnels de type flexible (Kuznik, 2014).

\section{Conclusion et perspectives de recherche}

Comme nous l'avons vu dans cet article, les trois disciplines scientifiques connexes que sont l'ergonomie, et plus concrètement l'ergonomie organisationnelle, la sociologie du travail et la traductologie partagent le même objet d'étude (le travail) et la même méthode de recherche (l'observation).

Inspirée par les intérêts et la méthodologie de recherche propres à la sociologie du travail, notre définition du contenu du travail des traducteurs internes a dû s'élargir à toutes les activités annexes qui, prises dans la durée totale du travail des traducteurs internes, en constituent la majeure partie : gestion de la sous-traitance et de toutes les perturbations survenues au cours du travail (Kuznik \& Verd, 2010), activités effectuées à l'aide d'un ordinateur (révision, gestion terminologique, gestion de la messagerie électronique, entre autres) ou sur support papier (révision, terminologie, par exemple) et en interaction avec d'autres employés du bureau de traduction (réunions de travail, fêtes, vie sociale; Kuznik, 2010), implication directe dans le processus de production et d'échanges économiques plus large (vente d'une machine dans le secteur alimentaire, par exemple) qui permet au traducteur d'avoir une vision globale de son activité de traduction proprement dite (Kuznik, 2016).

51 Ces résultats confirment d'ailleurs les résultats obtenus par Hébert-Malloch (2004): le traducteur expert qu'elle observe dans un contexte organisationnel précis (le Bureau de la traduction du Gouvernement du Canada), ne consacre que $41 \%$ de son temps de travail à la traduction proprement dite, la majeure partie de ce temps (59\%) étant accaparé par d'autres activités.

52 L'image du contenu du travail des traducteurs internes si vaste et hétérogène résultant de ces recherches nous fournit une évidence empirique et donc justifiée pour définir le poste de travail du traducteur interne, l'un des possibles profils des métiers traduisants, d'une manière ample et inclusive, telle qu'elle a été évoquée au début de la section 2 «Ergonomie appliquée à l'étude des pratiques de traduction: état de l'art». Par conséquent, une nouvelle question en découle : à savoir si, sous l'influence des pratiques et besoins sociaux nouveaux, la définition de la traduction proprement dite a évolué et s'approche de la définition et de la classification très larges proposées par Gottlieb (Gottlieb, 2008), dans lesquelles les conceptions de la traduction interlinguistique, intralinguistique et intersémiotique (Jakobson, 1959) sont totalement mélangées. Dans un futur projet de recherche, nous nous orienterons vers l'étude empirique de l'activité de 
traduction en tant qu'activité de service. Nous chercherons à savoir si, par un potentiel d'innovation élevé, cette conception élargie de la traduction s'accompagne d'un enrichissement de l'offre de services de traduction des prestataires en Pologne, et plus concrètement dans la ville de Wrocław, au sud-ouest du pays. La première étape de ce projet, qui constitue l'étude pilote, s'est déroulée dans la ville de Grenoble, avec la participation de l'UGA et avec le soutien financier du gouvernement français ${ }^{2}$.

Cette recherche vise à contribuer, à son tour, au développement de l'ergonomie organisationnelle, par une meilleure compréhension du sens et de la place de l'activité de traduction proprement dite au sein des entreprises prestataires de services de traduction dans deux pays européens.

\section{BIBLIOGRAPHIE}

ABDAlLAH Kristiina \& KoSKINEN Kaisa (2007), « Managing Trust: Translating and the Network Economy », Meta: Translators' journal, 52(4), 673-687.

Chesterman Andrew (2009), « The Name and Nature of Translator Studies », Hermes (42), 13-22, < download1.hermes.asb.dk/archive/download/Hermes-42-2-chesterman_net.pdf>.

Cicourel Aaron V. (2002), « La gestion des rendez-vous dans un service médical spécialisé », P. Bourdieu (dir.), Actes de la recherche en sciences sociales : Médecins, patients et politiques de santé, Broché, 143, 3-17.

COLler Xavier \& GARVía Roberto (2004), Análisis de organizaciones, Madrid, Espagne : Centro de Investigaciones Sociológicas.

DURAND Jean-Pierre (2000), « Temps, travail informationnel et flux tendu », C. Durand \& A. Pichon (dir.), Temps de travail et temps libre, Bruxelles : De Boeck.

DURAND Jean-Pierre (2004), La Chaîne invisible. Travailler aujourd'hui : flux tendu et servitude volontaire , Paris, France : Éditions du Seuil.

EHRENSBERGER-DOW Maureen (2014), «Challenges of translation process research at the workplace ", MonTI - Monographs in Translation and Interpreting, 1(7), 355-383, <dx.doi.org/10.6035/ monTI.2014.ne1.12>.

Falzon Pierre (2004), Ergonomie, Paris, France : Presses universitaires de France.

FERREIRA-AlVEs Fernando (2011), « Job Perceptions, Identity-Building and Interpersonal Relations among Translators as a Professional Group in Northern Portugal », ILCEA (14), < ilcea.revues.org/1119>.

FlyVbJERG Bent (2004), « Cinco malentendidos acerca de la investigación mediante los estudios de casos ", Reis (106), 33-62, <www.redalyc.org/articulo.oa?id=99717667002>.

FoEDISCH Melanie (2015, mars) « Translation Professionals as Networkers: An empirical study of quality in professional translation ", communication présentée au colloque Traducteurs à l'œuvre: approches ergonomiques des pratiques professionnelles et des formations de traducteurs, Grenoble, France. 
GotTLIEB Henrik (2008), « Multidimensional Translation », A. Schjøldager, H. Gottlieb \& I. Klitgård (dir.), Understanding Translation, Aarhus, Danemark : Academica, 39-65.

GouAdEC Daniel (2002), Profession : Traducteur, Paris, France : La Maison du Dictionnaire.

GouADEC Daniel (2005a), « Modélisation du processus d'exécution des traductions », Meta :journal des traducteurs, 50(2), 643-655.

GOUADEC Daniel (2005b) « Modèle unifié d'exécution de prestations de traduction/traducteur avec applications à la pratique professionnelle et à la formation des traducteurs ", Meta : journal des traducteurs, 50(4), <dx.doi.org/10.7202/019844ar>.

GouAdec Daniel (2007), Translation as a Profession, Amsterdam/Philadelphie : John Benjamins.

GRASS Thierry (2011), « "Plus" est-il synonyme de "mieux" ? Logiciels commerciaux contre logiciels libres du point de vue de l'ergonomie ", ILCEA (14), <ilcea.revues.org/1052>.

HÉBERT-MALLOCH Louise (2004), « What Do We Know About a Translator's Day », Meta: Translators' journal, 49(4), 973-979.

JAKOBSON Roman (1959) « On linguistic aspects of translation », R. A. Brower (dir.), On Translation, Cambridge : Harvard University Press, 233-239.

Jerome, Jerome Klapka (2006), 14 Books in 1, Londres : Shoes and Ships and Sealing Wax Ltd.

KosKINEN Kaisa (2008), Translating Institutions. An Ethnographic Study of EU Translation, Manchester, UK \& Kinderhook (NY), USA : St. Jerome Publishing.

KUZNiK Anna (2007a), « Les “tâches de traduction” en tant qu'indicateurs de la compétence de traduction dans une approche comportementale », D. Gouadec (dir.), Traduction, terminologie, rédaction: actes des Universités d'été et d'automne 2006 et du colloque international « Traduction spécialisée : quelle qualification universitaire pour les traducteurs?", Paris : La Maison du Dictionnaire, 117-132.

KUZNIK Anna (2007b), Estudio exploratorio sobre tareas traductoras (rapport de recherche non publié), Université Rennes 2 - Haute Bretagne, Rennes, <www.recercat.cat//handle/2072/9189>.

KUZNIK Anna (2008), « La investigación sobre el ámbito laboral del traductor », L. Pegenaute, J. De Cesaris, M. Tricás \& E. Bernal (dir.), La traducción del futuro: mediación lingüística y cultural en el siglo XXI, Barcelone : Promociones y Publicaciones Universitarias, 2, 69-379, <www.aieti.eu/pubs/ actas/III/AIETI_3_AK_Investigacion.pdf>.

KUZNIK Anna (2010), El contenido de los puestos de trabajo de los traductores. El caso de los traductores internos en las empresas de traducción de Barcelona (thèse de doctorat), Universitat Autònoma de Barcelona, Barcelone, <tdx.cat/handle/10803/5279>.

KuZNIK Anna (2011), « Puestos de trabajo híbridos. Cuatro indicadores del carácter heterogéneo de los puestos de trabajo internos en traducción », Sendebar (22), 283-307, <revistaseug.ugr.es/ index.php/sendebar/article/view/355/387>.

KUZNIK Anna (2014), « Translation as a Paradigmatic Universal, Post-Industrial, Knowledge-Based and Innovative Service ", inTRAlinea.online translation journal, Challenges in Translation Pedagogy, < www.intralinea.org/specials/article/2098>.

KUZNIK Anna (2015, mars), « Ergonomie organisationnelle. La place et le sens de l'activité de traduction dans les processus de travail dans les compagnies de traduction polonaises à Wrocław ", communication présentée au colloque Traducteurs à l'œuvre : approches ergonomiques des pratiques professionnelles et des formations de traducteurs, Grenoble, France. 
KUZNIK Anna (2016), « Work content of in-house translators in small and medium-sized industrial enterprises. Observing real work situations ", JoSTrans. The Journal of Specialised Translation, 25(1), 213-231, <www.jostrans.org/issue25/art_kuznik.pdf>.

KUZNIK Anna (à paraître) « Expérience d'un travail coopératif institutionnel avec les acteurs économiques du marché. Projet pilote de traduction à l'Institut d'Études Romanes de l'Université de Wrocław ", E. Monti \& P. Schnyder (dir.), Traduire à plusieurs. Collaborative Translations. Anciens défis et nouveaux enjeux, Paris : Orizons.

KUZNIK Anna \& VERD Joan Miquel (2010), « Investigating Real Work Situations in Translation Agencies.Work Content and its Components », Hermes (44), 25-43, < download2.hermes.asb.dk/ archive/download/Hermes-44-kuznik\&verd.pdf>.

LEBTAHI Yannicke \& IBERT Jérôme (2004), « Traducteurs dans la société de l'information.

Évolutions et interdépendances », Meta :journal des traducteurs, 49(2), 221-235.

LAVAULT-OlLÉON Élisabeth (2011a), « Une introduction à la problématique "Traduction et Ergonomie" ", ILCEA(14), <ilcea.revues.org/1118>.

LAVAULT-OLLÉON Élisabeth (2011b), «L'ergonomie, nouveau paradigme pour la traductologie », ILCEA(14), <ilcea.revues.org/1078>.

LAVAULT-OlLÉON Élisabeth (2011c), « Traduction et Ergonomie », ILCEA(14), <ilcea.revues.org/1031 $>$

LozAres Carlos, Verd Joan Miquel, Moreno Sara, BARRAnco Oriol \& Massó Matilde (2004), «El proceso de trabajo desde las perspectivas de la actividad situada y del conocimiento socialmente distribuido ", Cuadernos de Relaciones Laborales, 22(1), 67-87.

MayoRAl AsEnsio Roberto (2001), Aspectos epistemológicos de la traducción, Castellón de la Plana, Espagne : Publicacions de la Universitat Jaume I.

MiLls Charles Wright (1987), La imaginación sociológica, Barcelone, Espagne : Herder.

Monzó NeBOT Esther (2006), « ¿Somos profesionales? Bases para una sociología de las profesiones aplicada a la traducción », A. Parada \& O. Diaz Fouces (dir.), Sociology of translation, Vigo, Espagne : Servizo de Publicacións da Universidade de Vigo, 155-176.

MonZó Neвот Esther (2011), « Legal and translational occupations in Spain. Regulation and specialization in juristictional struggles », R. Sela-Sheffy \& M. Shlesinger (dir.), Identity and Status in the Translational Professions, Amsterdam/Philadelphie : John Benjamins, 11-30.

PENEFF Jean (1992), L’hôpital en urgence, Paris, France : A.-M. Métailié.

PENEFF Jean (1998), « Medida y control de las observaciones en el trabajo de campo. El ejemplo de las profesiones del sector servicios », Sociología del Trabajo (33), 3-25.

RISKU Hanna (2002), « Situatedness in Translation Studies », Cognitive Systems Research, 3(3), 523-533.

RISKU Hanna (2004), Translationsmanagement. Interkulturelle Fachkommunikation im Informations zeitalter, Tübingen : Narr.

RISKU Hanna (2014), « Translation process research as interaction research: From mental to socio-cognitive processes », MonTI. Monografías de Traducción e Interpretación, 1, 331-353.

RisKu Hanna \& Wimmer Petra (2006), « Integrating Technical Communication into Strategic Information Management », tcworld (6), 17-19. 
RISKU Hanna \& DiCKINSON Angela (2009), « Translators as Networkers: The Role of Virtual Communities », Hermes (42), 49-70, <download1.hermes.asb.dk/archive/download/Hermes-42-4risku\%26dickinson_net.pdf>.

RISKU Hanna, DicKInSON Angela \& PIRCHER Richard (2010), « Knowledge in Translation Studies and translation practice. Intellectual capital in modern society ", D. Gile, G. Hansen \& N. K. Pokorn (dir.), Why Translation Studies Matters?, Amsterdam/Philadelphie : John Benjamins, 83-96.

RISKU Hanna \& WindHAGER Florian (2013), « Extended Translation. A Socio-cognitive Research Agenda », Target. International Journal of Translation Studies, 25(1), 33-45.

RoSENSTIEL Lutz von (2004), « Organizational Analysis », U. Flick, E. von Kardoff \& I. Steinke (dir.), A Companion to qualitative research, Londres, Thousand Oaks, New Delhi : Sage Publications, 129-136.

Roy Donald (1952) « Quota Restriction and Goldbricking in a Machine Shop », American Journal of Sociology, 57(5), 427-442.

RYAN Richard (2011), «Traduction technique : les langues contrôlées au service de l'ergonomie documentaire », ILCEA (14), <ilcea.revues.org/1090>.

SKIBIŃSKA Elżbieta \& BLUMCZYŃSKI Piotr (2009), « Polish metaphorical perceptions of the translator and translation », Target. International Journal of Translation Studies, 21(1), 30-57.

TERSSAC Gilbert de (1992), Autonomie dans le travail, Paris : Presses Universitaires de France.

TOUDIC Daniel \& de BRÉBISSON Guillaume (2011) « Poste du travail du traducteur et responsabilité : une question de perspective », ILCEA (14), <ilcea.revues.org/1043>.

VANDAL-SIRoIS Hugo (2011), « Publicités multilingues : l'apport du traducteur en agence de communication marketing », ILCEA (14), <ilcea.revues.org/1106>.

VERD Joan Miquel, BARRANCO Oriol \& MORENO Sara (2007), «El análisis de los procesos de trabajo mediante métodos etnográficos: el caso del trabajo administrativo de consultas externas hospitalarias », Papers. Revista de Sociologia (83), 145-168.

VERD Joan Miquel \& LóPEZ Pedro (2008), « La eficiencia teórica y metodológica de los diseños multimétodo », EMPIRIA. Revista de Metodología de Ciencias Sociales (16), 13-42.

WOLF Michaela \& FUKARI Alexandra (2007), Constructing a Sociology of Translation, Amsterdam/ Philadelphie : John Benjamins.

\section{NOTES}

1. À partir de janvier 2016, suite à un processus de fusion, l'université Stendhal a été intégrée dans l'Université Grenoble Alpes (UGA).

2. Bourse pour un séjour de recherche d'un mois compris entre le 14 novembre et le 14 décembre 2015. 


\section{RÉSUMÉS}

Dans cet article, nous présentons certains points communs entre trois disciplines scientifiques connexes : l'ergonomie, organisationnelle notamment, la sociologie du travail et la traductologie. Ces trois disciplines partagent le même objet d'étude (le travail), les méthodes de recherche mises en œuvre (l'observation) et les difficultés particulières liées aux exigences de la recherche menée sur le terrain, au sein d'organisations à but lucratif : les entreprises. En effectuant une revue bibliographique nous donnons des exemples des principales études effectuées dans chaque domaine et nous démontrons comment elles ont contribué à la création de notre nouveau projet de recherche interdisciplinaire.

In this paper we present several aspects common to three interconnected scientific disciplines, namely: ergonomics, organisational ergonomics in particular, sociology of work and translation studies. These disciplines can share the same object of study (work), the same research method (observation) and specific difficulties linked to the demands of field work carried out in for-profit organisations, i.e. businesses. On the basis of a bibliographic review I provide examples of major studies performed in each of the disciplins in question and show how they have helped me develop a new interdisciplinary research project.

\section{INDEX}

Mots-clés : traduction, travail, contenu du travail, traductologie, ergonomie organisationnelle, sociologie du travail

Keywords : translation, work, work content, translation studies, organisational ergonomics, sociology of work

\section{AUTEUR}

\section{ANNA KUZNIK}

Université de Wroclaw 